\title{
$\Omega$
}

Ruth Sobala, Kevin Conroy, Hilary Tedd, Salem Elarbi

(1)

Respiratory Dept, Queen Elizabeth Hospital, Gateshead, UK.

\section{Eosinophils and effusion: a clinical conundrum}

\section{Case report}

A 60-year-old civil servant presented to the respiratory department with a 4-week history of dry cough, dyspnoea, rigours and malaise. She was a nonsmoker with a past medical history of well-controlled asthma, diagnosed 30 years previously. She had no pets. Medication consisted of a salbutamol inhaler only. On examination, chest sounds were vesicular and a general inspection was normal. She had no rashes, evidence of arthropathy or focal neurology. Routine blood tests revealed an eosinophilia of $9 \times 10^{9}$ eosinophils per $L$ and a $C$-reactive protein concentration of $106 \mathrm{mg} \cdot \mathrm{L}^{-1}$. Chest radiography and cross-sectional computed tomography (CT) imaging demonstrated consolidation of the right lower lobe with a moderate-sized pleural effusion (figures 1-3).

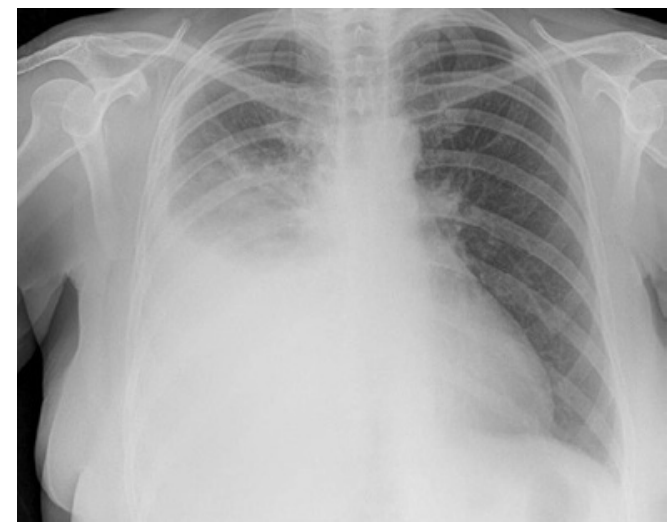

Figure 1 Initial chest radiography.

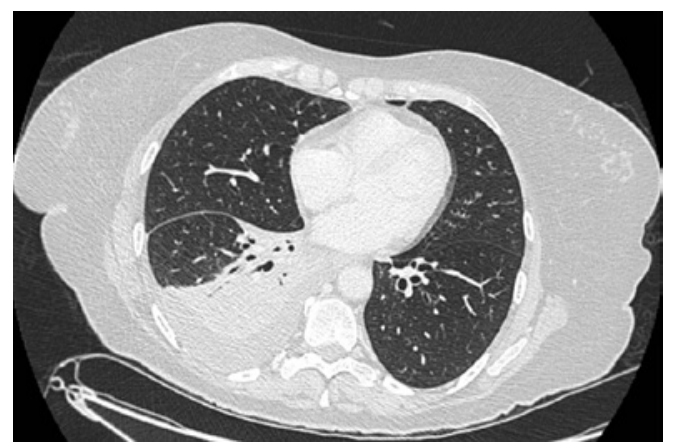

Figure 2 CT of the thorax demonstrating right-sided basal consolidation and effusion.

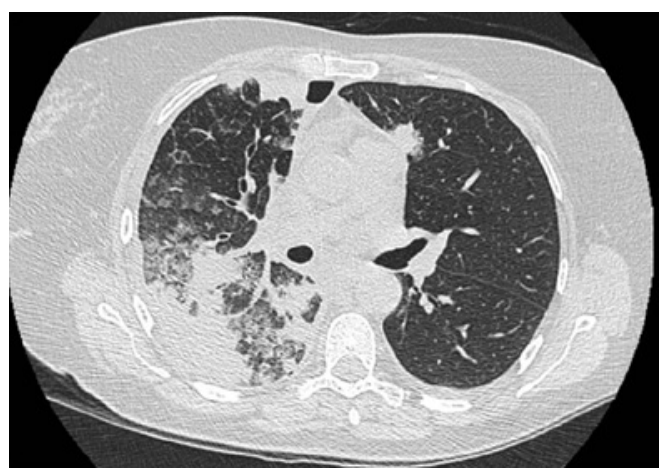

Figure 3 CT of the thorax demonstrating right-sided multifocal consolidation and effusion.

Task 1

What would be the most appropriate next step in investigating this patient?
a. Bronchoscopy with bronchoalveolar lavage $(B A L)$
b. CT-guided lung biopsy
c. Bone marrow biopsy
d. Thoracentesis with fluid analysis

Cite as: Sobala R, Conroy K, Tedd $\mathrm{H}$, et al. Eosinophils and effusion: a clinical conundrum. Breathe 2017; 13: e109-e113. 


\section{Answer 1}

d. Thoracentesis with fluid analysis. Whilst bronchoscopy is an important investigative step to exclude infection, haemorrhage and malignancy, management of the pleural effusion should take precedence. Relieving dyspnoea will make bronchoscopy easier and safer. Furthermore, a diagnosis could be made by analysis of the pleural fluid, meaning bronchoscopy would no longer be required. CT-guided lung biopsy and bone marrow biopsy will not relieve dyspnoea, and have no role in the initial investigation of this patient.

The right pleural effusion was drained of $1500 \mathrm{~mL}$ exudate via a Rocket therapeutic aspiration kit (Rocket Medical, Washington, UK). Samples sent for analysis demonstrated a marked eosinophilia, no malignant cells and no growth on routine culture.

\section{Task 2}

Which of the following is not a likely differential diagnosis for chest radiograph infiltrates and blood eosinophilia?

a. Hypereosinophilic syndrome

b. Asthma with allergic bronchopulmonary aspergillosis

c. Eosinophilic granulomatous with polyangiitis

d. Acute eosinophilic pneumonia 


\section{Answer 2}

d. Acute eosinophilic pneumonia. Elevated blood eosinophil count is typical of allergic or hypersensitivity disease, parasitic infections, and cancer. Acute eosinophilic pneumonia is not a likely differential diagnosis as typically this presents with a normal blood eosinophil count but sputum or BAL eosinophilia. The other differential diagnoses can all present with peripheral blood eosinophilia and radiographic changes.
Our patient developed progressive dyspnoea and chest radiography confirmed a worsening, moderate-sized pleural effusion. Arrangements were made for a Seldinger chest drain to be inserted. The patient had no history of foreign travel or change in medication. Anti-neutrophil cytoplasmic antibody (ANCA) and myeloperoxidase (MPO)/proteinase 3 titres were ordered to help rule out eosinophilic granulomatosis with polyangiitis. These returned as negative. Total IgE and Aspergillus-specific radioallergosorbent testing were also negative.

\section{Task 3}

In what proportion of patients with eosinophilic granulomatosis with polyangiitis would you expect to have a raised MPO/ perinuclear ANCA titre?
a. $0-1 \%$
b. $20-30 \%$
c. $40-60 \%$
d. $100 \%$ 


\section{Answer 3 \\ c. $40-60 \%$ [1].}

Following drainage of the pleural effusion via a chest drain, we performed repeat imaging of the chest. This demonstrated complete resolution of the pleural effusion. However, there was new multifocal consolidation, ground-glass opacification and intralobular septal thickening present throughout the right hemithorax (figure 4). We performed bronchoscopy and BAL. This showed a high number of respiratory epithelial cells with reactive changes but no eosinophilia in the lavage fluid. Endobronchial biopsies showed no evidence of malignancy.

\section{Diagnosis}

We commenced our patient on high-dose oral prednisolone and within 10 days she experienced near complete resolution of symptoms and chest radiograph opacities (figure 5). 6 months later, she remained well and had a normal eosinophil count, having completed a tapering course of prednisolone.

\section{Discussion}

There is a plethora of conditions associated with eosinophilic lung disease and accurate diagnosis can be difficult. Careful history taking and clinical examination are crucial to detect signs and clues in order to make a diagnosis. In the absence of exotic travel or recent medication changes, we must be careful to exclude vasculitides or haematological conditions such as hypereosinophilic syndrome or malignancy [2, 3]. Along with a negative MPO test the absence of worsening asthma symptoms, rash or other extrapulmonary symptoms are able to point us away from eosinophilic granulomatosis with polyangiitis [1, 2]. Hyper eosinophilic syndrome is also classically seen with organ-specific features, such as diarrhoea and urticaria, and raised IgE [4]. This is also effectively ruled out. The absence of lymphadenopathy on cross-sectional imaging, and otherwise normal blood film and cytogenetic testing, make underlying malignancy unlikely [4]. This led us to a clinical diagnosis of chronic eosinophilic pneumonia.

Chronic eosinophilic pneumonia is a rare condition with an unknown aetiology thought to be due to dysfunction of interleukin 5 [5]. It most commonly affects nonsmoking females in middle age, with a high proportion of patients describing a history of asthma. Classically, it will present with chronic cough, progressive dyspnoea, and constitutional symptoms such as weight loss and night sweats [2, 4]. Extrapulmonary symptoms

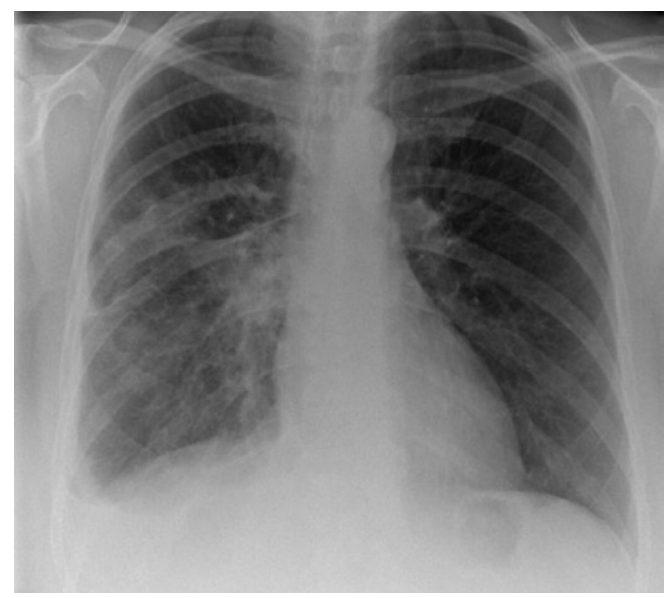

Figure 4 Chest radiography following removal of the Seldinger chest drain.

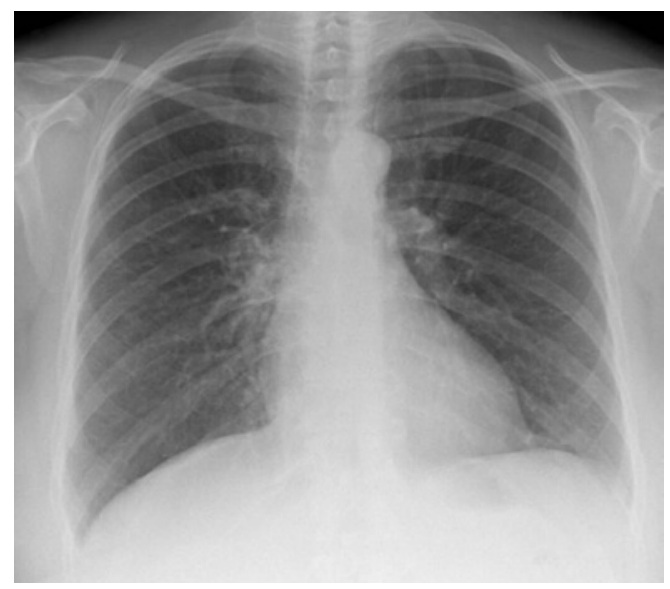

Figure 5 Chest radiography demonstrating resolution of airspace shadowing following 10 days of prednisolone.

are very rare. It is a clinical diagnosis based on the presence of respiratory symptoms for $>2$ weeks, pulmonary infiltrates, BAL and/or peripheral blood eosinophilia, and exclusion of other causes of eosinophilic lung disease [2, 4].

Classically, imaging will demonstrate multiple areas of consolidation described as a "photo negative of pulmonary oedema", though multiple findings have been described, such as reticulation and nodules, and pleural effusions have been reported in up to $10 \%$ of patients in case series $[2,4,6]$.

BAL should demonstrate a marked eosinophilia $(>40 \%)[2,7]$, although this was not seen in our patient. We believe that prednisolone therapy introduced prior to the bronchoscopy may have obfuscated the overall clinical picture. Despite this, our patient's symptoms and serum laboratory findings would all be consistent with chronic eosinophilic pneumonia. Her rapid clinical and radiological response is also classical for chronic eosinophilic pneumonia [2]. 


\section{Conflict of interest}

None declared.

\section{References}

1. Hirohata S. Differential characteristics of MPO-ANCA positive and negative eosinophilic granulomatosis with polyangiitis. J Allergy Clin Immunol 2015; 135: Suppl., AB135.

2. Marchand E, Cordier J-F. Idiopathic chronic eosinophilic pneumonia. Orphanet J Rare Dis 2006; 1: 11.

3. Hayakawa $\mathrm{H}$, Sato $A$, Toyoshima $M$, et al. A clinical study of idiopathic eosinophilic pneumonia. Chest 1994; 1462-1466.

4. Jeong YJ, Kim KL, Seo IJ, et al. Eosinophilic lung diseases: a clinical, radiologic, and pathologic overview. Radiographics 2007; 27: 617-637.
5. Alam M, Burki NK. Chronic eosinophilic pneumonia: a review. South Med J 2007; 100: 49-53.

6. Luks AM, Altemeier WA. Typical symptoms and atypical radiographic findings in a case of chronic eosinophilic pneumonia. Respir Care 2006; 51: 764-767.

7. Marchand E, Reynaud-Gaubert M, Lauque D, et al. Idiopathic chronic eosinophilic pneumonia. A clinical and follow-up study of 62 cases. The Groupe d'Etudes et de Recherche sur les Maladies "Orphelines" Pulmonaires (GERM“O”P). Medicine (Baltimore) 1998; 77: 299-312 